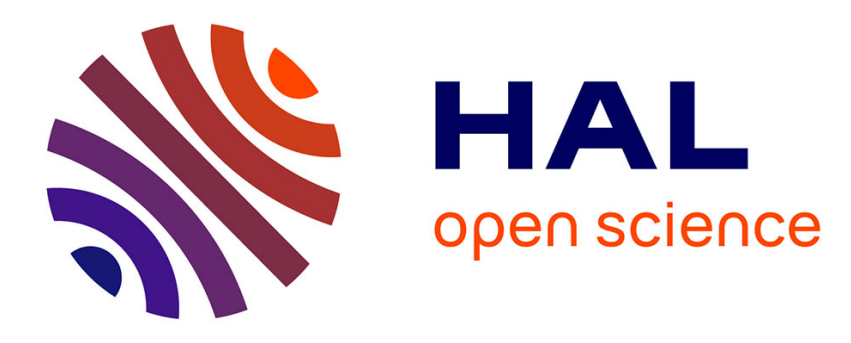

\title{
La parole hyperbolique en interaction: une figuralité entre soi-même et même
}

Bertrand Verine

\section{To cite this version:}

Bertrand Verine. La parole hyperbolique en interaction: une figuralité entre soi-même et même.

Langue française, 2008, Figures et point de vue, 160, pp.117-132. 10.18463/verine.002 . hal-01314719

\section{HAL Id: hal-01314719 \\ https://hal.science/hal-01314719}

Submitted on 11 May 2016

HAL is a multi-disciplinary open access archive for the deposit and dissemination of scientific research documents, whether they are published or not. The documents may come from teaching and research institutions in France or abroad, or from public or private research centers.
L'archive ouverte pluridisciplinaire HAL, est destinée au dépôt et à la diffusion de documents scientifiques de niveau recherche, publiés ou non, émanant des établissements d'enseignement et de recherche français ou étrangers, des laboratoires publics ou privés. 


\title{
LA PAROLE HYPERBOLIQUE EN INTERACTION : UNE FIGURALITÉ ENTRE SOI-MÊME ET MÊME
}

\author{
Bertrand Verine, CNRS - Université Montpellier 3, Praxiling UMR 5267
}

[Ce travail a été présenté à la journée Conscila du 19 octobre 2007, Figures et point de vue (Rabatel A. dir.). Une version étendue a paru dans Langue française $\mathrm{n}^{\circ} 160$, p. 117-131. Les nombres intercalés dans le texte sans parenthèses ni crochets indiquent la pagination des segments communs avec la version papier.]

Mais le peuple vautour, / Au bec retors, à la tranchante serre, / Pour un chien mort se fit, dit-on, la guerre. / Il plut du sang : je n'exagère point. La Fontaine, « Les vautours et les pigeons ».

Les recherches praxématiques conduites dans le cadre de l'équipe Praxiling s'assignent pour horizon d'élaborer une linguistique de la parole et une méthodologie d'analyse textuelle. À ce double titre, Jeanne-Marie Barbéris et Jacques Bres ont, dès les années 1980, fait le pari d'articuler les genres premiers du discours ordinaire et les genres seconds de l'oral préparé ou de l'écrit savant. Plus encore, dès l'origine, Robert Lafont et Françoise Gardès-Madray (1976/1989 : 108) ne considèrent pas les figures comme «utilisées ou non ponctuellement », mais comme « une fonction toujours à l'œuvre dans le langage ».

La description praxématique de cette fonction figurale n'ayant, à ce jour, été appliquée qu'au processus métaphorique par Catherine Détrie (2001), je remercie Alain Rabatel de me donner l'occasion de tenter une approche du fait hyperbolique ${ }^{1}$. Je définirai celui-ci comme la construction d'une évaluation ${ }^{2}$ de l'objet du discours plus intense que celle ordinairement associée à la représen-

118

tation d'objets comparables. Après avoir justifié cette définition en déport de celles proposées par la rhétorique récente, je montrerai l'intérêt qu'il peut y avoir à subsumer l'hétérogénéité des indices linguistiques d'évaluation intensive sous un point de vue (désormais PDV) hyperbolisant que le locuteur articule plus ou moins polémiquement avec le PDV dominant dans l'interdiscours, et plus ou moins empathiquement avec le PDV du/des destinataires.

\section{Pour des hyperboles vives et heureuses}

\subsection{Une exécrable querelle}

En préambule, je ne résiste pas au plaisir de citer deux points de vue agonaux dont les sociotypes caricaturaux tombent dans le ridicule qu'ils entendent prévenir :

- Cette figure est la ressource des petits esprits qui écrivent pour le bas peuple (...) Mais quand on a du génie et de l'usage du monde, on ne se sent guère de goût pour ces sortes de pensée fausses et outrées (Port-Royal apud Dumarsais, 1731/1988: 133);

- Elle est dans la bouche de tout le monde, mais surtout des jeunes gens (soucieux de passer pour des héros) et des jeunes filles (soucieuses de faire valoir leur exceptionnelle sensibilité). (...) l'hyperbole est une figure d'une écœurante facilité. C'est elle qui dévalorise le style (Morier, 1961 : 495-496).

De telles charges explicitent les soupçons diffus de mauvais goût et d'abus de langage qui parasitent la problématique des hyperboles. Le soupçon de vulgarité se dénoue pour ainsi

\footnotetext{
${ }^{1}$ Envisagé ici pour lui-même, donc indépendamment de l'ironie avec laquelle il interagit dans bien des cas.

${ }^{2}$ Je n'entrerai pas dans la distinction classique entre auxèse méliorative et tapinose péjorative.
} 
dire de lui-même : théorise-t-on la créativité du processus métaphorique d'après le seul exemple d'un travail de cochon, ou sa cohérence discursive d'après Le char de l'État navigue sur un volcan (Henri Monnier) ? Plus sérieusement, cette défiance permet d'introduire le lien des hyperboles au contexte sous la double espèce des genres du discours et de la réception sociohistorique. Si leurs contempteurs de la fin du XVII ${ }^{\mathrm{e}}$ ou du $\mathrm{XX}^{\mathrm{e}}$ siècles les restreignent aux genres datés et situés du discours ordinaire, de la presse ou de la publicité, Catherine Kerbrat-Orecchioni (1994) pointe leur opérativité dans la politesse mondaine. Du côté des genres valorisés de l'oral préparé ou de l'écrit, Dumarsais lui-même (ibidem) reconnaît leur présence non seulement «dans le style satirique et badin », mais «quelquefois même dans le style sublime et poétique »; Olivier Reboul (1991 : 62) montre leur efficacité argumentative dans les genres épidictique et judiciaire, très ancrés dans le contexte ; Joëlle Gardes-Tamine et Marie-Claude Hubert (1993 : 11) soulignent leur rôle dans la fatrasie médiévale, Jean-Jacques Robrieux (2000:93) leur caractère de «pilier du langage précieux » et de «figure clé du romantisme », dont les œuvres ont connu une fortune fluctuante.

La diversité de ces états de service n'empêche pas que, plus qu'aucune autre figure, l'hyperbole semble avoir à se défendre contre le soupçon de faire des phrases qui, en bonne logique, devrait peser sur l'ensemble du pathos, voire de la rhétorique : alors qu'un discours abondamment ou malencontreusement

119

métaphorique sera dit, avec commisération, fleuri, toute parole hyperbolique paraît au risque d'être stigmatisée pour son emphase, son exagération, son excès ou sa grandiloquence. C'est avec ces quatre termes que le Grand Robert met en réseau son entrée hyperbole, et si sa définition échappe à l'axiologie en citant Marouzeau ("mettre en relief une idée " au moyen d'une expression qui la dépasse " »), il enregistre par la synonymie une tendance historique lourde. De fait, l'excès, qui n'apparaît qu'à la marge chez Lamy et Dumarsais, devient un circonstant définitoire pour Fontanier et Littré, avant de se transformer en exagération, presque toujours nominalisée, de Larousse à nos jours :

- L'hyperbole est un trope qui représente les choses ou plus grandes, ou plus petites qu'elles ne sont dans la vérité. On emploie les hyperboles lorsque les termes ordinaires sont ou trop faibles, ou trop forts ; et qu'ils ne se trouvent pas proportionnés à notre idée : ainsi craignant de ne pas dire assez, on dit plus (...) On pardonne cet excès ; parce qu'en se servant de termes ordinaires, on ne dirait pas assez (Lamy 1715/1998 : 202) ;

- Lorsque nous sommes vivement frappés de quelque idée que nous voulons représenter, et que les termes ordinaires nous paraissent trop faibles pour exprimer ce que nous voulons dire, nous nous servons de mots qui, à les prendre à la lettre, vont au-delà de la vérité, et représentent le plus ou le moins pour faire entendre quelque excès en grand ou en petit. Ceux qui nous entendent rabattent de notre expression ce qu'il en faut rabattre, et il se forme dans leur esprit une idée plus conforme à celle que nous voulons y exciter, que si nous nous étions servis de mots propres (Dumarsais 1731/1988:131);

- l'hyperbole augmente ou diminue les choses avec excès, dans le but de fixer, par ce qu'elle dit d'incroyable, ce qu'il faut réellement croire (Fontanier 1821/1968:123);

- augmenter ou diminuer excessivement la vérité des choses pour qu'elle produise plus d'impression (Littré) ;

- figure par laquelle on avance une proposition très-exagérée, pour produire sur l'esprit une forte impression (Larousse) ;

- écart par rapport au jugement pondéré : exagération (on dit le plus pour le moins) (Morier 1961 : table) ;

- l'hyperbole (...) est non seulement une façon exagérée d'exprimer sa pensée, mais aussi la forme extrême de l'argument de direction, qui réfute une thèse en disant : si on l'admet, jusqu'où va-t-on (Reboul 1991: 219) ;

- discours amplifiant la réalité (...) acte de langage d'exagération (...) énonciation excessive (Bonhomme $2005: 103) \ldots$

Seuls, à ma connaissance, Jacques Dubois et al. (1970:134), peut-être parce qu'ils ne s'attardent guère sur les hyperboles, s'abstiennent de toute axiologisation : " on " augmente " les choses c'est-à-dire qu'on modifie les sèmes intensifs ». Pour sa part, Georges Molinié (1992 : 166) parle d'exagération dans le commentaire d'un exemple, mais reste neutre dans sa définition : 
une hyperbole est une figure qui joue sur la caractérisation intensive d'une information ; (...) on dit plus que la valeur « véritable » du contenu. (...) le simple énoncé de chacun des groupes, assorti d'un vocabulaire fortement 120

intensif, voire redondant dans l'intensité, oblige le lecteur à traduire chaque fois à un degré plus bas de désignation.

\subsection{Comment tout embrasser sans étreindre trop mal ?}

En filigrane des jugements socio-esthétiques d'intempérance par rapport au bon ton et d'infraction au dogme du mot juste, ces définitions révèlent l'embarrassante nécessité non seulement de prendre ensemble le locuteur, le récepteur, le message et le référent, mais de modéliser le processus même de leur interaction. Dans une lecture paradigmatique attentive aux constantes, toutes semblent se rejoindre sur la difficile obligation de sortir de l'immanence de la langue en prenant en compte, si peu que ce soit, le référent, les choses (Lamy, Fontanier, Littré, Dubois et al.) ou l'information (Molinié), voire la subjectivité du locuteur, son idée (Dumarsais), son jugement (Morier), sa pensée (Reboul). De proche en proche, le fait hyperbolique insécurise même le rapport du discours à la réalité (Fontanier, Bonhomme), voire à la vérité (Lamy, Dumarsais, Littré, Molinié) : de même qu'un locuteur ironique peut toujours être soupçonné de railler, outre l'objet de son discours, son destinataire, le locuteur hyperbolisant semble toujours susceptible de tromper son allocutaire, à l'instar de l'enfant blessé ou chagrin que ses parents suspectent d'en rajouter. Ainsi Bonhomme (2005 : 144-145) distingue-t-il, selon leurs paramètres véridictoires et référentiels, les souscatégories du discours littéral extrême, de l'hyperbole appropriée, de l'hyperbole non appropriée et de l'hyperbole mensongère.

Il est vrai que, dans la plupart des occurrences à l'exception de certains adynatons du type un bruit à réveiller les morts ${ }^{3}$, ce n'est pas la signification des mots qui se trouve déréglée / reréglée, mais le mode de référenciation de l'énoncé qui semble subverti. Cependant, pour pouvoir en juger de manière systématique, il faudrait disposer d'une description de la normativité représentationnelle qui, plus que l'énoncé lui-même, détermine selon Jean-Claude Chevalier l'image d'expérience que se forge le récepteur d'un discours : « des multiples représentations que je peux me construire d'une même réalité il en est une qui l'emporte parce que, à tort ou à droit, réputée plus directement adéquate à ladite " réalité " : c'est ce que j'ai proposé d'appeler l'orthologie » (Chevalier et Delport, 1995 : 103). Or, si ce concept est particulièrement opératoire pour rendre compte des écarts de traduction qui passent effectivement par la substitution d'un énoncé à un autre, force est de constater que nous ne possédons pas même les principes d'une telle description.

Une lecture diachronique attentive à la variation est peut-être susceptible d'aider à sortir de cette aporie. Elle montre que c'est dans le cadre de linguistiques opposant un usage informatif et un usage ornemental du langage qu'on en est venu à focaliser les définitions de l'hyperbole sur une prétendue distorsion manipulatrice visant à produire plus d'impression que la vérité des choses (Littré) - ce qui, par décalages successifs, conduit Molinié à faire porter le

121

travail de traduction du récepteur non plus sur la caractérisation, mais sur la désignation de l'objet du discours. Au contraire, Lamy posait comme une des situations discursives possibles la non-coïncidence entre l'évaluation du locuteur et l'évaluation orthologiquement associée au référent par les termes ordinaires : « ou trop faibles, ou trop forts (...) ils ne se trouvent pas proportionnés à notre idée ». Dumarsais précisait même que

\footnotetext{
${ }^{3}$ Je n'entre pas dans le débat entre la définition faible d'hyperbole impossible et la définition forte de proposition combinant l'hyperbole, la comparaison et l'apodioxe (réfutation par l'absurde).
} 
l'hyperbole permet à l'esprit du récepteur de se former « une idée plus conforme à celle que nous voulons y exciter, que si nous nous étions servis de mots propres ». Très symptomatiquement, aussi bien Lamy (op. cit. : 208) que Dumarsais (op. cit. : 133) signalent comme favorisant la félicité de l'hyperbole des commentaires métaénonciatifs pointant le degré d'adéquation du discours à son objet: non seulement les défensifs pour ainsi dire, si l'on peut parler ainsi ou si j'ose me servir de ces termes, mais le revendicatif pour m'exprimer plus hardiment.

Dans cette lignée et dans le cadre ici tracé par Alain Rabatel, mon hypothèse de travail consistera à réintégrer de manière non axiologique le PDV du locuteur et son interrelation dynamique, explicite ou implicite, avec un, voire plusieurs PDV autres, associables à l'interdiscours ou au destinataire. Je proposerai donc (i) de définir comme hyperboliques les énoncés construisant une évaluation de l'objet du discours plus intense que celle ordinairement associée à la représentation orthologique d'objets comparables; (ii) de rechercher les marques du dialogue interne à ces énoncés entre le PDV du locuteur et un PDV autre; (iii) de rapporter le sentiment d'insécurité référentielle des récepteurs de certains énoncés intensifs, non à l'hyperbole en tant que telle, mais à l'emploi par le locuteur hyperbolisant de formes sollicitant l'empathie des destinataires, qui relèvent d'une textualisation en même.

Je rappelle que la praxématique dénomme textualisation en soi-même la production (la plus fréquente, en particulier à l'écrit) d'énoncés construisant un spectacle linguistique ayant atteint le seuil d'objectivité dont le locuteur est la caution et que le locuteur articule explicitement, si besoin est, avec les énoncés (effectifs ou imaginés) de son destinataire ou de l'interdiscours. Par opposition, elle dénomme textualisation en même la production (notamment à l'oral ou dans la littérature expérimentale) d'énoncés supposant l'engagement praxique du destinataire dans une sphère expérientielle commune et construisant un spectacle linguistique basé sur des schémas d'expérience ou sur des représentations partagées impliquant la non-disjonction des PDV (cf. notamment Détrie et Verine 2003).

\subsection{De la dé-mesure en toutes choses, ou presque}

Rappelons rapidement les indices linguistiques de tous ordres permettant de repérer une séquence discursive probablement hyperbolique. Les marqueurs morphologiques d'intensité font consensus: affixes superlatifs du type -issime ou méga- ${ }^{4}$, comparatifs généraux de supériorité (articles définis suivis de plus),

122

tous les comparatifs simples non seulement de supériorité, mais d'égalité et d'infériorité selon le choix de leur complément ${ }^{5}$, adverbes marquant le haut degré en langue (beaucoup, très, trop) ou en discours (carrément, complètement) et dont la liste ne cesse de se renouveler (furieusement, méchamment).

Le reste du lexique pose avec une acuité renouvelée la question de la référenciation abordée supra. Aucun des ouvrages récents de lexicologie ne suit Robrieux (2000:93) dans sa tentation d'identifier des termes par nature hyperboliques, comme "catastrophique " ou "épouvantable". Quand il enregistre des emplois intensifs figés, le Robert les signale comme sens affaibli (j'ai horreur de ce temps) ou comme exagération (dans toute son horreur : avec tous ses aspects horribles et, par exagér., désagréables). On soulignera cependant l'abondance des clichés hyperboliques : par exemple, tous ceux recourant aux nombres trentesix, cent ou mille, et la très intéressante hypothèse diachronique de Ronald Landheer : "plus

\footnotetext{
${ }^{4} \mathrm{Ou}$ mini-, micro-, etc., quand ils portent à son comble, c'est-à-dire près du néant, un trait de faible intensité, comme peuvent également le faire les suffixes diminutifs dans d'autres langues romanes.

5 Ainsi quand un esclave sucrier amputé d'un bras et d'une jambe énonce : «Les chiens, les singes et les perroquets sont mille fois moins malheureux que nous » (Voltaire, Candide, XIX).
} 
un mot a un caractère fort ou polarisant, soit au niveau de la quantification, soit au niveau de l'affectivité, plus il sera sensible à un emploi hyperbolique qui l'oriente vers une polysémisation » $(2007: 136)$.

À côté de telles lexies, je propose donc de considérer comme indice lexical d'une possible séquence hyperbolique la co-occurrence en discours de mots constituant le plus haut ou le plus bas degré d'un paradigme gradable, de mots signifiant l'extrême généralisation (parmi lesquels les adverbes toujours vs jamais, les pronoms tout vs rien, les déterminants tout vs aucun) ou la singularité absolue (notamment seul, unique et leurs dérivés). La plupart des auteurs notent que ces choix lexicaux peuvent s'enrichir de tropes portant à son comble un trait de l'objet du discours : ainsi passe-t-on aisément du comparatif aussi rapide qu'une tornade à la comparaison rapide comme une tornade et à la métaphore une tornade passa, c'était X. Marc Bonhomme (1998: 57) leur ajoute les synecdoques généralisantes, en tant qu'elles provoquent «des grossissements de perspectives sur certaines entités, désignées par l'ensemble qui les englobe ».

La syntaxe est souvent négligée par les analystes, soucieux de limiter le fait hyperbolique aux formes isolables du groupe ou de la phrase simple. Or, dès qu'on a identifié la catégorie des superlatifs, il serait incohérent de ne pas la compléter par celles des structures consécutives de type si / tant/ tel ... que, assez / trop ... pour ou à + infinitif: cette dernière fournit d'ailleurs de nombreux clichés hyperboliques (une foi à déplacer les montagnes), non sans rester créative (aimer à perdre la raison, Aragon). De même, aussitôt qu'on a repéré l'expression lexicale de la singularité absolue, il est logique de lui adjoindre la restriction qui, du paradigme des possibles niés, excepte un élément unique, à l'instar de l'exemple de JeanBaptiste Rousseau cité par Fontanier (ibidem) : l'éternité pour moi ne sera qu'un moment. Comme précédemment, le répertoire des indices syntaxiques s'accroît de toutes les figures produisant un effet d'intensification

123

cumulative, au premier rang desquelles ${ }^{6}$ la réduplication et la gradation (Bonhomme 1998 : 46 et 76).

Un des intérêts de l'analyse en termes de PDV hyperbolisant est de donner un statut explicite à la cohérence discursive de ces éléments hétérogènes en langue. Leur inventaire provisoire semble d'ailleurs donner quelque crédit a priori à notre hypothèse de travail pour deux raisons. (i) À l'exception des choix lexicaux et des tropes associés, la grande majorité des indices d'un possible processus hyperbolisant impliquent par définition l'évaluation du locuteur: ainsi en va-t-il non seulement des superlatifs, comparatifs, adverbes intensifs et structures consécutives, mais encore des restrictions et de la plupart des négations en tant qu'elles présupposent un énoncé autre sur le même objet du discours. (ii) Plusieurs de ces indices ont été analysés comme marquant, de manières très différentes que je ne saurais détailler ici, la dialogisation interne de l'énoncé par les PDV effectifs ou supposés d'autres locuteurs-énonciateurs : aux négations et restrictions s'ajoutent sur ce point les comparatifs selon Jacques Bres (1998), les métaphores in praesentia et les synecdoques selon Détrie (2001 et 2006). En revanche, les indices déterminants que sont les superlatifs, les adverbes intensifs et les structures consécutives, ainsi que la majorité des choix lexicaux et les métaphores in absentia, ne portent pas trace en eux-mêmes d'un autre PDV et demandent à être interprétés en fonction de leur interaction cotextuelle avec les éléments précédents, voire avec certains paramètres référentiels quand ils sont accessibles ou remémorables. Il en va de même pour l'emploi discursivement congruent de certains indices prosodiques, que je proposerai (infra 2.) d'ajouter à cette liste ouverte.

\footnotetext{
${ }^{6}$ Je ne peux approfondir ici le lien que Dubois et al. (1970 : 136) établissent entre hyperbole et antithèse.
} 
$\mathrm{Au}$ total, j'insisterai sur deux tendances symétriques fondamentales. Un principe de différenciation, d'une part : à l'exception de quelques formes brèves, comme certains slogans publicitaires, pour que les indices hyperboliques produisent leur effet de mise en saillance, il n'est pas possible que le locuteur « regarde d'un même œil les petites et les grandes choses ; que tout lui paraisse grand; qu'il estime aussi bien une bagatelle, que la chose la plus sérieuse et la plus importante, et qu'il parle de tout avec un style égal » (Lamy op. cit. : 208). Un principe de cohésion, d'autre part: les fragments prototypiquement hyperboliques n'adviennent presque jamais au discours de but en blanc et sans postérité, mais s'inscrivent dans un réseau intensif orientant la production-réception d'une séquence. C'est (entre autres arguments) à ce titre que Dubois et al. (1970:123-136) font de l'hyperbole un métalogisme, Reboul (1991:129 et 160) un trope complexe, Molinié (1992:39 et 166) une figure macrostructurale de second niveau. Réciproquement, l'emploi conventionnel de termes intensifs à la mode, tels que super ou génial pour la période récente, ne suffit pas pour qu'on puisse catégoriser l'énoncé hôte comme hyperbolique. En dehors de quelques cas limites ${ }^{7}$ à analyser en fonction du genre discursif et du contexte, l'équilibre

124

entre ces deux principes de différenciation et de cohésion paraît indispensable à la félicité de la parole hyperbolique.

\section{La construction interactionnelle du sens hyperbolique}

Je n'appliquerai provisoirement ces hypothèses qu'à des occurrences d'hyperboles heureuses, celles que l'interlocuteur ne court-circuite ni par une injonction négative du type [n'en fais pas trop quand même], ni par des mises en doute plus ou moins explicites équivalant à [tu n'en fais pas un peu trop là ?]. Je travaillerai sur trois fragments d'interactions spontanées recueillies à micro caché ${ }^{8}$ : propos de table entre amis de longue date en vacances dans le village de Conil en 1998, et conversations dans un salon de coiffure de quartier depuis 2006.

\subsection{La connivence hyperbolique contrariée par l'exactitude référentielle Soit l'exemple [1]:}

[1] (Après le récit d'une rencontre déplaisante que vient de faire Marianne, notée C, la conversation porte sur la manière expressive que cette locutrice a de faire la gueul' gueul' ou gueul' numéro 1.)

$21 \mathrm{C}$ - je n' peux pas tu sais (sélectionne B) c'est plus fort que moi / je suis euh complèt'ment primair' mais si j' / si quèqu' chos' me fâche me vexe ou: / si quelqu'un me plaît pas / (1) je fais la gueul' comme un gosse (rire) je bous dans mon coin je ne peux pas le cacher je fais rir' tout l' monde c'est complèt'ment ridicul' je ne peux pas le cacher (1) / j' suis là / (coup de glotte bouche fermée) / ou alors quelqu'un qui me déplaît ou: je (3) je ne peux pas supporter le mec euh (3) / Laur' Pougnal $\uparrow$ tu t'en souviens euh Tristan $\uparrow$

22A - oui:

$23 \mathrm{C}$ - je ne peux pas supporter Jacques Pougnal mais je ne peux pas

$24 \mathrm{~A}$ - on l'a jamais connu lui

$25 \mathrm{C}$ - ah ben vous avez du bol / d'abord il a l'horrible manie de tripoter les gens (ha ha A) tu sais i ne peut pas te parler (oui A) sans te: euh toucher l'épau:le:

$26 \mathrm{~B}$ - ah j'ai horreur de ça

$27 \mathrm{C}$ - ouh là

28B - ah e:t

$29 \mathrm{C}-$ alors je

\footnotetext{
${ }^{7}$ Cf. par exemple la publicité intégralement hyperbolique pour Rivella analysée par Bonhomme (2005 : 103 104) et, inversement, l'adynaton Ici la boue est faite de nos pleurs démarquant le PDV d'Agathe par rapport à la modération du poème « Maesta et errabunda » de Baudelaire.

${ }^{8}$ Conventions de transcription : [:] allongement vocalique; [/] pause ; ['] apocope ; [个] intonation ascendante ; l'encadrement d'un segment par [(1)] indique une inflexion rieuse, par [(2)] un ton élevé, par [(3)] un ton affaibli. Tous les noms désignant des personnes privées ont été remplacés par des pseudonymes.
} 
30B - les cheveux aussi les homm's ou les gens qui te trifougn'nt (je / je je je je: C) les cheveux

$31 \mathrm{D}-$ ah bon $\uparrow$

$32 \mathrm{~B}-\mathrm{a}: \mathrm{h}$

33D - ça exist' ça $\uparrow[. .$.$] (Conil IV).$

\section{5}

Les tours 21 et 23 présentent de nombreux indices de la construction par la locutrice $\mathrm{C}$ d'une évaluation hyperbolique de sa propension à afficher le renfrognement sur son visage. On y relève en effet le comparatif de supériorité c'est plus fort que moi, la double occurrence de l'adverbe intensif complèt'ment, la métaphore lexicalisée je bous et la sextuple répétition du noyau phrastique je ne peux pas : la première en construction absolue, les quatre suivantes soulignées prosodiquement par des accents de groupe et par l'absence d'élision sur le discordantiel ne, la dernière, également en emploi absolu, renforcée par l'utilisation dérivée du coordonnant mais à des fins de renchérissement. Nous observerons (infra 2.2.) que c'est par la triple reprise de je ne peux pas que Marianne conduira la séquence à son terme. Entre temps, son propos va connaître une interruption de $2 \mathrm{mn} 52 \mathrm{~s}$ occupées par 27 tours de parole. La méconnaissance par le locuteur A d'un des référents visés par l'énoncé semble être la cause première de ce détour de la conversation : on l'a jamais connu lui (tour 24).

$[\ldots]$

126

\subsection{La textualisation hyperbolique, entre soi-même et même}

C'est seulement au tour 53 de la même conversation que Marianne parvient à clore son propos et à réinstaurer son PDV hyperbolisant :

[2] (La même locutrice narre une sortie de fin d'année scolaire à laquelle se sont joints de nombreux enseignants extérieurs, dont le collègue précédemment en cause.)

52B - yavait plus d'adultes que d'élèves en fin d' compte

53C - ouais / ouais / en fait c'est ça // e:t je trouv' ça crapuleux pasque c'est un voyag' d'élèves je vois pas pourquoi les adultes euh viendraient et surtout je trouv' crapuleux qu'on me foute euh (dans les pattes A) (1) Pougnal que j' peux pas blairer dans les pattes / alors j' me suis installée dans l' bus / j'ai croisé mes p'tits bras j'ai fait la moue et ça a duré comm' ça trois heures / et alors (1) ya la copin' qui v'nait euh / ça va t'as pas mal au cœur non non ça va non non / (1) non ça m'a trop fait chier chier chier chier (1) / après j' me suis tirée pour pas aller bouffer avec lui au pub mais vraiment la sal' goss' mais je / je ne / peux pas je n' peux pas me taper le mec Pougnal plus de un quart d'heur' sa:ns / voilà /// (Conil IV).

Aussi bien dans ce tour que dans les tours 21, 23 et 25 (supra), la locutrice construit globalement la séquence hyperbolique selon une textualisation en soi-même opposant son évaluation à celle d'autres instances. Avant d'en observer les marques, il convient de souligner qu'en conversation ce choix est indépendant de l'existence, ou non, d'une connivence préalable entre les interactants. Celle-ci ne fait en l'occurrence aucun doute, comme l'ont montré les éléments relevés supra et comme le confirment ici les anticipations des interlocuteurs : Marianne appuie son discours sur la proposition de B yavait plus d'adultes que d'élèves en fin d' compte, qu'elle confirme et anaphorise par ouais / ouais // en fait c'est ça / et je trouv' ça, puis sur le groupe dans les pattes interpolé par A après qu'on me foute euh, qu'elle intègre à la syntaxe de sa phrase. Le choix du mode de textualisation paraît avant tout lié au projet discursif du locuteur : si toute séquence hyperbolique suppose un fort degré d'implication, on a ici affaire à une confidence humoristique dans laquelle l'engagement intime se trouve mitigé par l'autodérision.

Le dialogisme antagonique propre à la textualisation en soi-même peut être interphrastique et séparer clairement les PDV. Ainsi au tour 25 Marianne représente-t-elle le même procès selon son évaluation hyperbolique, il a l'horrible manie de tripoter les gens, 
puis selon le PDV de l'actant générique te, voire du délocuté lui-même : il ne peut pas te parler sans te: euh toucher l'épau:le:.

\section{7}

De même, au tour 21, articule-t-elle par mais deux évaluations intensives contradictoires de sa propre attitude : je suis euh complèt'ment primair' mais (...) je fais la gueul' comme un gosse je bous dans mon coin. C'est notamment cet antagonisme qui conduit à interpréter l'emploi intensif de complèt'ment et le cliché je bous non comme des manières de dire stéréotypées, mais comme participant d'une hyperbole vive. On peut en dire autant, au tour 53, de la proposition non dialogisée ça m'a trop fait chier chier chier chier, que l'ensemble du cotexte permet de recevoir non comme un abus de langage, mais comme contribuant, par l'adverbe intensif trop et la triple réduplication de chier, au PDV hyperbolisant de la locutrice.

La dialogisation s'avère plus fréquemment intraphrastique, marquant l'antagonisme avec un PDV autre sans expliciter son origine. Ainsi la double occurrence de crapuleux (tour 53), manifestement intensive pour évaluer des invitations indues, est-elle introduite en fonction d'attribut du COD par je trouve qui, en posant de manière antagonique le PDV de la locutrice, présuppose l'existence d'au moins un autre PDV. Il en va de même du comparatif c'est plus fort que moi (tour 21), renchérissant sur des évaluations dépréciatives du type [c'est un peu fort] ou [c'est trop fort] pour mieux asserter le caractère incoercible du comportement de Marianne. C'est enfin le cas avec la modalisation je ne peux pas qui, certes, n'a rien d'intensif en soi, mais se trouve contextuellement mise au service du PDV hyperbolique en martelant cette même irrépressibilité et en réfutant les PDV autres [elle pourrait cacher son désappointement] puis [elle pourrait supporter son collègue]. La conclusion de la séquence rend ce dispositif particulièrement frappant en combinant des formes inter- et intraphrastiques : mais vraiment la sal' goss' mais je / je ne / peux pas / je n' peux pas.

Par opposition, on doit rapporter à la textualisation en même le cœur du tour 21 où la locutrice juxtapose sans les identifier clairement ni les hiérarchiser des propositions dont, seul, le cotexte large montre qu'elles correspondent à des PDV différents : je ne peux pas le cacher je fais rir' tout l' monde c'est complèt'ment ridicul' je ne peux pas le cacher. L'opacité de ce fragment laisse (furtivement) aux interlocuteurs la responsabilité active d'établir les connexions du sens et de s'orienter empathiquement dans la polyphonie des PDV au lieu d'assister à leur objectivation par le discours. Sans doute peut-on en dire autant de la suspension finale, apparemment délibérée puisque la locutrice amorce un groupe prépositionnel non indispensable à la phrase qu'elle clôt aussitôt, ainsi que son tour de parole : je n' peux pas me taper le mec Pougnal plus de un quart d'heur' sa:ns / voilà. Dès lors, la pause entre sa:ns et voilà fonctionne comme une sorte de muet [vous savez quoi] auquel les interlocuteurs ne peuvent assigner un contenu qu'en adoptant le PDV de Marianne (sauf à lui demander d'expliciter ou à se désengager de l'interaction).

\subsection{Un exemple de séquence hyperbolique tendant vers la textualisation en même}

Il en va beaucoup plus massivement ainsi dans l'exemple [3] :

\section{8}

[3] (Dans un salon de coiffure mixte, plusieurs des cinq participants ne se sont pas rencontrés depuis longtemps. Au cours de l'échange de nouvelles, Lisa, notée A, explique pourquoi elle souffre de fringale.)

$1 \mathrm{~A}$ - je viens de passe:r deux ans de catastrophes j'ai perdu beaucoup de monde: euh jeune // donc ça a agi sur mon poids /// derrière j'ai été cocue neuf mois // ça m'a: / perturbée // mais l'amour va mieux ////// donc j'ai eu un mea culpa de quelques mois là j'en sors / je reprends du poil de la bête

$2 \mathrm{~B}$ - oh ça se voit hein

3A - mais c'est long quand même eh je trouve eh / puis parfois ya des moments eu:h j'ai des pics t' sais j'ai des montées encore de mauvaise f- / de mauvaises pensées quoi (hm B) et puis cette aberration qui est qui me lâche pas quoi //// ce que je voudrais qui me lâche c'e:st euh Satan / par moments je vais bien je vais bien je vais bien 
tout d'un coup j'ai des pics / des pics de Satan Satan arriv' dans ma bouche je deviens eu:h / méchante / (3) méchante (3) / c'est ma mère qui me l'a fait remarquer / ell' m'a dit ma fille je te reconnais plus tu deviens méchante / mais après ça me passe quoi ça m' ça va me durer une heur' deux heures tu vois mai:s / j'emmerde mon monde et là plus personne vient me voir / sauf vous (rire A et D) //// des fois je te dis pas la salope / infernale eh j'en ai voulu au (2) monde entie:r (2) // tu sais c'est toujours le genr' de chose:s / les autres mais pas moi quoi ///

$4 \mathrm{~B}$ - alors ça /// ça

$5 \mathrm{~A}$ - puis moi j’avais Moïse eh

4B - la Bible le dit que quand ça te pre:nd euh

5A - moi j'avais Moïse dans les bras quoi alors tu sais euh //// (2) e:nfin (2) / et puis j'ai dix ans de (2) moins (2) / ça aussi ç'a été pour moi:: //// l'aberration /// puis i souffrait de rien quoi / enfin on croit que toujours que tout va bien e:t /// (Salon de coiffure 19.8.2006).

Cet exemple confirme plusieurs des observations précédentes. D'une part, la situation référentielle sur laquelle porte l'évaluation intensive est initialement posée en termes explicites : j'ai été cocue neuf mois // ça m'a: / perturbée. D'autre part, la locutrice fait le choix de l'hyperbole et d'un mode de textualisation empathique en fonction de son projet discursif et en dépit de conditions peu propices : certes, le salon de coiffure est fréquemment le lieu d'épanchements intimes, mais la communication apparaît ici compliquée par le bruit parasite du sèche-cheveux et par la multiplicité des destinataires qui n'ont pas tous le même degré de familiarité entre eux ni avec Lisa. Or, si les quatre occurrences de $t$ 'sais, tu sais ou tu vois privilégient le contact avec l'interlocutrice B, la précision sauf vous ratifie les autres participants en tant que destinataires de la séquence hyperbolique.

À l'instar des exemples [1] et [2], cette séquence contient quelques traits de textualisation en soi-même. Ainsi la locutrice recourt-elle à certains clichés intensifs, immédiatement interprétables comme tels par les récepteurs, non sans les raviver par leur cotexte large, par leur juxtaposition syntagmatique et par l'élévation du ton dont elle affecte le dernier: je te dis pas la salope / infernale eh j'en ai voulu au monde entie:r. De même, la spécificité du PDV de Lisa se trouve-t-elle posée de manière antagonique en une occurrence au moyen de pour moi, et articulée dialogiquement aux PDV de trois autres instances. On relève en premier

129

lieu le double discours rapporté, indirect, c'est ma mère qui me l'a fait remarquer, puis direct, ell' $m$ 'a dit ma fille je te reconnais plus tu deviens méchante : il pointe la proposition je deviens méchante comme l'expression du PDV extérieur et normatif sur le procès que, dans sa perspective, Lisa vient de décrire par j'ai des pics / des pics de Satan Satan arriv' dans ma bouche. On retrouve ensuite l'exception sauf vous, qui signale le caractère hyperbolique de la généralisation plus personne vient me voir, avec pour double effet de prévenir une atténuation consolatrice du type [mais si nous venons te voir] et de retrancher les destinataires de la catégorie des autres pour les poser en mêmes. Enfin, je te dis pas fonctionne conventionnellement, dans ce type de contextes, à la fois comme prétérition et comme commentaire métaénonciatif qui, en exhibant le jeu paradoxal entre dire et ne pas dire, renforce la prédication la salope, et la soustrait à toute discussion.

Cependant, ces éléments de textualisation en soi-même apparaissent en milieu de séquence et s'avèrent moins déterminants pour le processus hyperbolisant que les choix lexicaux et les tropes in absentia induisant la réception empathique des destinataires. On doit rapporter à ce dernier type de fonctionnement le réglage de sens idiosyncrasique de mea culpa dans j'ai eu un mea culpa de quelques mois là j'en sors : l'insertion syntaxique hors norme (avoir un), la durée de quelques mois, incompatible avec le sens dictionnairique, et le cotexte plus large semblent devoir orienter le récepteur vers un réglage du type [phase d'intense autoculpabilisation, de contrition profonde]. Les deux occurrences d'aberration apparaissent beaucoup plus opaques. La première est actualisée au moyen du démonstratif cette, sans 
qu'on puisse l'associer avec certitude à aucun élément du cotexte amont ou aval. La seconde est mise en équivalence avec la proposition j'ai dix ans de moins par la structure attributive ça aussi ç'a été pour moi:: //I/ l'aberration, mais son actualisation au moyen de l'article défini l' ne laisse pas de surprendre en conférant au terme une extension absolue, et le connecteur aussi demande de catégoriser comme relevant de cette même aberration un ou plusieurs éléments du cotexte amont sans qu'aucune autre instruction ne vienne guider les calculs inférentiels du récepteur. Plus encore, aussi bien le thème associé à la seconde occurrence que la relative qui me lâche pas $^{9}$ déterminant la première contredisent les acceptions les plus fréquentes d'erreur d'appréciation ou d'égarement cognitif au profit, semble-t-il, de celle d'absurdité. On constate ainsi que le dérèglement du mode de référenciation n'est pas imputable au PDV hyperbolisant en tant que tel, mais au questionnement induit par certains des choix lexicaux auxquels recourt la locutrice pour le construire.

Un processus comparable est induit par les trois emplois de Satan, dans la mesure où le pseudo-clivage ce que je voudrais qui me lâche c'e:st incite à recevoir ce nom comme un rhème inédit et dissuade de l'identifier comme la cause des pics ou montées de mauvaises pensées qui ont déjà été mentionnés : sauf à refuser toute connivence et à se désintéresser de la conversation, le récepteur se trouve conduit, par le questionnement interprétatif ainsi ouvert, à épouser le PDV

130

hyperbolisant de Lisa au moins jusqu'à ce que la reformulation conventionnelle je deviens méchante vienne stabiliser le réglage métonymique du sens. Il en va de même, enfin, pour la proposition j'avais Moïse dans les bras, que rien ne prépare dans le cotexte amont et avec laquelle le cotexte aval ne construit que deux liens des plus ténus : en l'absence de complément du comparatif $j$ 'ai dix ans de moins ${ }^{10}$ et de tout autre nom masculin dans le cotexte, il est loisible de supposer que Moïse puisse constituer, d'une part, le terme étalon implicité et, d'autre part, le référent du pronom sujet dans $i$ souffrait de rien. De telles inférences ont pu être favorisées par les connaissances préalables des destinataires sur la locutrice : tous savent, en particulier, qu'aucun homme de son entourage n'a pour prénom Moïse. Cela m'a personnellement conduit, en tant qu'observateur participant, à conclure qu'il s'agissait probablement d'une métaphore in absentia par laquelle Lisa verbalise la représentation d'innocence au-dessus de tout soupçon qu'elle avait de son mari avant de découvrir son infidélité. Il convient cependant de souligner l'absence de cohésion textuelle de ce fragment et le fait que, beaucoup plus que le résultat du calcul interprétatif, c'est le processus même qui est ici pertinent, en ce qu'il conduit à nouveau les destinataires à adopter le PDV hyperbolisant de la locutrice.

Ce fonctionnement n'est pas vérifiable par les réactions des interlocuteurs qui, de fait, apparaissent moins nombreuses et moins significatives que dans les exemples [1] et [2] : le régulateur $h m$ du début du tour 3 intervenant en limite initiale du processus, on ne dispose guère que du rire synchronisé avec celui de la locutrice dans la fin du même tour et, surtout, de l'enchaînement sémantique par la Bible dans la seconde partie du tour 4. Cela n'a rien d'étonnant en soi, car l'adhésion empathique a par définition moins besoin de se dire que l'accord de PDV posés comme disjoints mais convergents; de plus, quand l'énoncé construisant l'empathie est, comme ici, particulièrement opaque, on peut supposer les interlocuteurs plus occupés par leur travail de réception et moins disponibles pour anticiper leur réponse. L'interlocutrice $\mathrm{B}$ fait donc preuve d'une forte réactivité empathique en tentant de prolonger par la Bible le réseau, sémantiquement frappant mais syntagmatiquement lâche, constitué par mea culpa, Satan, infernale et Mö̈se.

\footnotetext{
${ }^{9}$ La construction de ce verbe avec un sujet abstrait est elle-même tropologique.

10 Tous les participants savent que la locutrice est moins âgée que son mari, mais il n'a pas encore été explicitement question de lui, et il peut également s'agir de sa rivale.
} 
Au terme de ce parcours, le fait hyperbolique demeure délicat à cerner à cause de son caractère fondamentalement discursif, de la diversité de ses marques linguistiques et de la pluralité de ses utilisations interactionnelles. En particulier, les pistes présentées ici demandent à être éprouvées sur des occurrences mettant de surcroît en œuvre un PDV ironique, ou dans des genres discursifs beaucoup plus stéréotypés comme les mondanités, voire seconds comme la publicité. Par contre, une telle approche ne doit poser aucun problème dans les textes littéraires, à condition que les jeux avec l'interdiscours et la hiérarchisation entre auteur, narrateur et actants soient bien repérés, ce qui n'est pas le cas chez tous les analystes consultés pour ce travail.

131

En raison de cette diversité, l'analyse en termes de points de vue paraît apte à favoriser une approche unifiante du phénomène. Dans les séquences étudiées ici, elle permet de confirmer que l'essentiel du processus hyperbolisant ne porte pas sur le référent mais sur son évaluation, et que l'insécurité de la référenciation relevée par de nombreux auteurs n'est pas le propre du fait hyperbolique, mais de la textualisation en même, que le locuteur peut solliciter pour stimuler l'empathie des destinataires.

C'est sans doute ce recours qui apparaît très marqué - voire déplacé ou intrusif à certains analystes - dans les genres de l'oral préparé et de l'écrit où le destinataire est, par définition, moins en phase avec le locuteur. Au contraire, dans les genres de l'oral spontané, la construction d'une connivence empathique semble généralement ressentie comme gratifiante et, si elle ne l'est pas, le destinataire reste toujours libre de la court-circuiter en revenant, par une demande de précision, à la textualisation en soi-même. Pour en juger, il convient désormais de recueillir un corpus de séquences hyperboliques incluant des malentendus ou des dissensus.

\section{Références}

Bonhomme M., 1998, Les Figures clés du discours, Paris : Seuil.

Bonhomme M., 2005, Pragmatique des figures du discours, Paris : Champion.

Bres J., 1998, "Vous les entendez? Analyse du discours et dialogisme », Modèles linguistiques XX/2, 71-86.

Chevalier J.-C. \& Delport M.-F., 1995, L'Horlogerie de saint Jérôme (problèmes linguistiques de la traduction), Paris : L'Harmattan.

Détrie C., 2001, Du sens dans le processus métaphorique, Paris : Champion.

Détrie C., 2006, «La production de sens synecdochique : relation partitive et/ou phénomène de saillance ? », in Kleiber G., Schnedecker C. \& Theissen A. (éd.), La Relation "partietout », Louvain : Peeters, 783-797.

Détrie C. \& Verine B., 2003, «Modes de textualisation et production du sens : l'exemple de " Complainte d'un autre Dimanche " de Jules Laforgue », in Amossy R. \& Maingueneau D. (dir.), L'Analyse du discours dans les études littéraires, Toulouse : P.U. du Mirail, 213-225.

Dubois J. et al., 1970, Rhétorique générale, Paris : Larousse.

Dumarsais C., 1731/1988, Des tropes ou des différents sens, Paris : Flammarion.

Fontanier P., 1821/1977, Les Figures du discours, Paris : Flammarion.

Gardes-Tamine J. \& Hubert M.-C., 1993, Dictionnaire des figures littéraires, Paris : Armand Colin.

Kerbrat-Orecchioni C., 1994, «Rhétorique et pragmatique : les figures revisitées », Langue française 101, 57-71.

Lafont R. \& Gardès-Madray F., 1976/1989, Introduction à l'analyse textuelle, Montpellier : Service des Publications de l’Université Paul-Valéry.

Lamy B., 1715/1998, La Rhétorique ou l'art de parler, Paris : Champion. 
Landheer R., 2007, «Polysémie et polysémisation sous l'effet de l'emploi hyperbolique », in Trotter D. (éd.), Actes du XXIV congrès international de linguistique et de philologie romanes (Aberystwyth 2004), vol. IV, Tübingen : Max Niemeyer Verlag, 129-138.

Molinié G., 1992, Dictionnaire de rhétorique, Paris : Poche.

Morier H., 1961, Dictionnaire de poétique et de rhétorique, Paris : PUF.

Reboul O., 1991, Introduction à la rhétorique, Paris : PUF.

Robrieux J.-J., 2000, Rhétorique et argumentation, Paris : Armand Colin. 